\title{
The Diagnostic Validity of the Criteria for Binge Eating Disorder
}

\author{
Janet D. Latner, $\mathrm{PhD}^{1 *}$ \\ Courtney Clyne, $\mathrm{PhD}^{2}$
}

\author{
ABSTRACT \\ Objective: This paper considers whether \\ the criteria currently used to classify the \\ diagnosis of binge eating disorder (BED) \\ are valid and appropriate. \\ Method: We review evidence that \\ reflects on the validity of the current cri- \\ teria for binge eating episodes and BED, \\ using literature retrieved through major \\ psychology and psychiatry search engines \\ (e.g., Psyclnfo, PubMed). \\ Results: Evidence from experimental \\ research points to the relative importance \\ of episode frequency, the amount of food \\ consumed at episodes, the subjective \\ sense of loss of control over eating, and \\ several additional criteria associated with \\ binge episodes in BED. Evidence on the \\ differences in psychopathology between
}

BED and bulimia nervosa and between BED and obesity without binge eating, as related to diagnostic criteria, is reviewed.

Conclusion: Although evidence concerning the diagnostic criteria of BED is mixed, broadening certain diagnostic criteria for binge eating episodes and BED might more accurately reflect the research literature and increase the number of individuals eligible for inclusion in treatment programs. (c) 2007 by Wiley Periodicals, Inc.

Keywords: binge eating; binge eating disorder; diagnostic criteria; objective bulimic episodes; subjective bulimic episodes; DSM

(Int J Eat Disord 2008; 41:1-14)
Binge eating disorder (BED) is highly prevalent among individuals seeking treatment for obesity, with prevalence estimates ranging between 15 and $50 \%$. In the general population, the lifetime prevalence of BED is between $0.7 \%$ and $4 \% .{ }^{1}$ BED is defined in the fourth edition (text revision) of the Diagnostic and Statistical Manual (DSM-IV-TR) as frequent binge eating episodes, characterized by the consumption of a large quantity of food in a discrete period of time, accompanied by a subjective sense of loss of control over eating. Individuals with BED must also experience distress about their binge eating, and binge episodes are associated with at least three of the following: eating more rapidly than normal, eating until uncomfortably full, eating large amounts when not hungry, eating alone because of embarrassment, and feeling disgusted, depressed, or guilty about overeating. ${ }^{1}$

\footnotetext{
Accepted 15 July 2007

*Correspondence to: Janet D. Latner, Department of Psychology, University of Hawaii at Manoa, 2430 Campus Road, Honolulu, HI 96822. E-mail: jlatner@hawaii.edu

${ }^{1}$ Department of Psychology, University of Hawaii, Honolulu, Hawaii

${ }^{2}$ Department of Psychology, University of Otago, Dunedin, New Zealand

Published online 5 October 2007 in Wiley InterScience (www.interscience.wiley.com). DOI: 10.1002/eat.20465 (C) 2007 Wiley Periodicals, Inc.
}

BED, as it is currently defined, is associated not only with eating disorder psychopathology, but also with comorbid psychiatric disorders, health problems, and obesity. ${ }^{2}$ Health-related quality of life is significantly impaired in obese women with BED relative to obese women without BED., ${ }^{3,4}$ For these reasons, it has been argued that BED is a distinct and significant disorder of clinical severity that requires specific treatment. ${ }^{5}$ However, it has also been proposed that the frequent psychiatric comorbidity, good response to many forms of treatment, and the lack of agreement on what constitutes a large amount of food make the BED diagnosis more useful as a marker for other psychopathology in obese individuals than as a clinical disorder in its own right. ${ }^{6}$

Another potential problem with the diagnostic definition of BED lies in the question of whether the criteria for the disorder are valid. Kendell and Jablensky ${ }^{7}$ have suggested that validity in psychiatric disorders is present if the defining characteristics of a syndrome can be demonstrated to be an entity, separated clearly from neighboring syndromes and from normality. This article examines the evidence regarding the DSM-IV criteria for BED. The diagnostic criteria have two major components: the definition of a binge episode, and BED-specific criteria. This review first discusses the 
validity of the criteria for a binge episode (drawing on evidence from both BED and bulimia nervosa (BN), since binge eating is a common and characteristic feature of these disorders). It then discusses the validity of the criteria specific to BED. To determine whether BED is clearly separate from neighboring syndromes and from normality, the distinctiveness of BED from BN is considered, as well as the distinctiveness of BED (which is often accompanied by obesity) from obesity that is not accompanied by frequent binge eating. Finally, this article considers some of the implications of the definition of BED for clinical practice, future research, and diagnostic systems.

\section{Defining Binge Eating}

The core symptom of BED is, arguably, binge eating itself. The criteria for the frequency of binge eating episodes required to meet a diagnosis of BED, however, and the most appropriate definition of a binge eating episode are important issues that have attracted a good deal of attention in recent empirical research. The criteria used to define binge eating episodes in the DSM-IV-TR include (1) the consumption of a large amount of food, (2) the perception of a loss of control over eating, and (3) the episode occurs within a discrete period of time. ${ }^{1}$ Research using diverse approaches has examined the suitability of each of these criteria. The defining criteria for binge episodes have important implications for the diagnosis and treatment of eating disorders. Broadening these criteria would lead to a diagnosis of BED in a greater number of individuals. Conversely, narrowing them would restrict the numbers that receive a diagnosis.

A DSM-IV-TR diagnosis of BED requires that binge eating must occur at an average of at least 2 days per week for at least 6 months for BED. ${ }^{1}$ For a diagnosis of $\mathrm{BN}$, binge eating episodes occur at an average of at least two occasions per week for at least 3 months. The difference in the requirement of frequency of days in BED and episodes in BN may be due to the more discrete nature of binges in $\mathrm{BN}$, where episodes are often terminated by compensatory behavior. (Nevertheless, binges are not to be defined by associated compensatory behavior.) In BED, episodes may have a more extended time frame, making it at times more appropriate to consider binge days, rather than binge episodes. On the other hand, the difference in duration between the two sets of diagnostic criteria has been questioned, as little research exists to inform this difference. Cooper and Fairburn ${ }^{8}$ have suggested that the current BED duration criterion (6 months) be brought in line with those of anorexia nervosa and BN (3 months).

\section{Large Amount of Food}

Fairburn and Cooper ${ }^{9}$ distinguished two basic types of binge eating episodes that often occur in individuals with eating disorders. Objective bulimic episodes (OBEs) involve the consumption of a large amount of food accompanied by a loss of control, and subjective bulimic episodes (SBEs) involve the consumption of a small to moderate amount of food accompanied by a loss of control. In the laboratory, individuals with BED typically consume well over 1,000 kcal during binge episodes. ${ }^{10}$ Individuals with BED consume more food at binges than weight-matched obese individuals without BED who are asked to binge eat. ${ }^{11,12}$ BED patients also consume a greater percentage of energy from fat, as well as more dessert and snack foods, than weight-matched controls instructed to binge. ${ }^{13}$

It is also important to determine whether individuals with BED use quantity of food to define their own binges. Some research has indicated that the amount of food eaten may be an important determinant of whether individuals with BED perceive their own eating episodes as binges. Support for the large quantity criterion comes from a study of eating-disordered and nondisordered participants asked to eat foods they felt were "forbidden" and those that were not "forbidden." ${ }^{14}$ In both the forbidden and nonforbidden conditions, both groups of participants were more likely to label their eating episode a binge as the quantity of food believed to be consumed increased. In another study on self-defined binges, 42 women with BED and 40 weight-matched controls were asked to rate the minimum amounts of eight foods they would consider to be "binges" and to identify the "largestever" servings they had consumed of foods, ${ }^{15}$ BED participants identified the "largest-ever" servings they had consumed as larger than those of controls for all foods, suggesting that these individuals may consume or binge on greater quantities of foods than their weight-matched counterparts. Those with BED had similar judgments to controls about how much food they considered to be a binge. These results are consistent with previous data that people with BED are not higher on dietary restraint as measured by the Eating Disorder ExaminationQuestionnaire (EDE-Q) ${ }^{16}$ than controls. ${ }^{17}$ Therefore, it might be unusual for individuals with BED to classify small eating bouts as binges by due merely to the "abstinence violation effect," the belief that having eaten only a small amount of a forbidden food constitutes failure or a binge. 
Instead, larger size might often be a defining characteristic of participant-defined binge episodes.

Other evidence suggests that many binge eaters may not use a large-size criterion to classify their own binges. Eating episodes labeled as binges in food diaries by women with BN were larger, on average, than episodes not labeled as binges. ${ }^{18}$ However, a high proportion of these episodes were in fact relatively small, despite still being labeled as binges. Similarly, Telch et al. ${ }^{19}$ found that only $43 \%$ of women with BED used large quantity as a criterion to define their own overeating episodes as binges (while $82 \%$ used the criterion of loss of control). Telch and Agras ${ }^{20}$ also found that it was not primarily the amount of food consumed, but rather negative mood and the experience of loss of control (discussed below), that determined whether or not participants labeled episodes as binge eating.

Research by Raymond et al. ${ }^{21}$ compared the energy intake of 12 women with BED on binge days (days when binge eating occurred) versus nonbinge days. They found a significantly greater number of calories consumed on investigator-defined binge days. This finding was consistent with previous research on the food intake of women with BED, showing greater food consumption on binge days than nonbinge days. ${ }^{22}$ Binge days also involved different eating patterns from nonbinge days, with more food consumed in the evenings on binge days relative to nonbinge days. ${ }^{21}$ However, these differences were significantly attenuated when participant-defined binges were used to designate binge days rather than this objective criterion. This discrepancy between investigator-defined and participant-defined binges suggests that individuals with BED may use a lower threshold than investigators (who used a criterion of $>1,000 \mathrm{kcal}$ to define binges) for the amount of food that they believe constitutes a binge.

An innovative research design directed actresses to eat in different scenarios that varied based on (1) the quantity of food eaten (large or small), (2) loss of control (present or absent), and (3) the duration of the episode (short or long). The authors examined whether these vignettes were judged to be binges by 23 participants with BED [defined as those meeting criteria for the disorder on the Questionnaire on Eating and Weight Patterns (QEWP) ${ }^{23}$ ], 34 clinical psychologists, and 25 university students without eating disorders. ${ }^{24}$ Episodes combining large quantity with short duration more often resulted in judgments that the episode resembled a binge. However, binge eaters were more sensitive to large quantities of food consumed, rating scenarios involving large quantities as more binge-like than university students without eating disorders. Compared to the professional group, binge eaters were also more likely to characterize large episodes accompanied by a loss of control as "weak" and "unacceptable." An earlier version of this study also found that the consumption of a large quantity of food did influence the likelihood of the episode being rated as a binge, but only when large quantity was combined with short time duration. ${ }^{25}$ These results also highlight the possible importance of quantity to binge eaters themselves in defining binge episodes.

The consumption of large quantities of food in BED may be related to physiological differences between obese individuals with and without BED. For example, obese individuals with concurrent BED were shown to have an increased gastric capacity relative to obese individuals without BED. $^{26,27}$ The gastric capacity of obese women with $\mathrm{BED}$ was similar to lean women with $\mathrm{BN}$, while the gastric capacity of obese women without BED was similar to lean controls. This enlarged capacity may be a result of binge eating, but it may also play a role in the perpetuation of binge eating by contributing to a deficient satiety response. ${ }^{28}$

Summary: It is clear that individuals with BED consume greater amounts of food at binge episodes than controls, in laboratory studies where controls are weight-matched and asked to eat as much as they can. Self-reported binge episodes, recorded through food diaries, are also often large. Binge eaters frequently use the size of an episode to determine whether their own or someone else's eating should be labeled a binge. However, self-labeled binge episodes are often not objectively large, suggesting that binge eaters also rely on other criteria in defining binge eating.

\section{Loss of Control}

In the assessment of loss of control, Cooper and Fairburn ${ }^{8}$ have emphasized that the binge eater needs to experience a "definite sense of loss of control at the time of eating, rather than retrospectively inferred lack of control" (p. S93). However, it is also clear that the decision about the experience of lack of control is a subjective one to be made by the individual who is eating. In the $\mathrm{EDE},{ }^{9}$ if interviewees answer in the affirmative to the question of whether or not they experienced a loss of control over eating, interviewers are instructed to take their word for it. Only when an interviewee answers in the negative is the interviewer instructed to proceed with further detailed questions probing the nature of the loss of control. 
The majority of research on the characteristics that define a binge eating episode supports the criterion stipulating that the subjective sense of loss of control over eating be present during the episode. Compared with responders with recurrent overeating (objective overeating episodes without a loss of control), those with BED showed more body image concerns and psychological distress. ${ }^{29}$ The finding suggests that loss of control (rather than eating large amounts) is a key determinant of psychological problems, and eating large amounts of food may be, in itself, relatively less relevant to psychopathology. Johnson et al. ${ }^{24,25}$ gathered ratings from noneating disordered participants on their beliefs about what constitutes a binge episode. In their research involving videotaped vignettes depicting actresses eating in scenarios varying the quantity of food eaten, loss of control, and the duration of the episode, noneating-disordered women and men watched the enactments and rated the degree to which they thought the episode was a binge. ${ }^{25}$ The effect for loss of control on binge ratings was independent of the other characteristics: episodes were considered more binge-like each time a loss of control was present. A follow-up to this study also found that the presence of a loss of control over eating also resulted in higher binge ratings by individuals with BED, psychologists, and healthy students. ${ }^{24}$ The findings of Johnson et al. provide overall support for all three DSM-IV-TR criteria for binge eating episodes (food quantity, loss of control, duration of episode), and especially strong support for the loss of control criterion. Similarly, $82 \%$ of women with BED used the criterion of loss of control to define their own binges. ${ }^{19}$

The perception of loss of control over eating may be affected by mood. In the study by Guertin and Conger discussed above, ${ }^{14}$ a negative mood induction increased ratings of loss of control among participants with high scores on the Bulimia Test (a reliable and valid self-report scale by which to identify individuals with BN). ${ }^{30}$ On the other hand, more positive mood enhanced ratings of self-control over eating. This finding suggests that while loss of control may be a valid criterion for defining binge eating, patients' reporting of this experience may be influenced by mood and thus may not always be accurate.

Pediatric research findings on the loss of control are consistent with the findings from adults. Among children aged 6-13, those who reported having had eating episodes involving a loss of control, regardless of the size of these episodes, were more likely to experience eating disordered cognitions than children without eating episodes involv- ing a loss of control. ${ }^{31}$ Similarly, 6- to 10 -year-old children who had experienced episodes of a loss of control over eating were heavier and had greater body dissatisfaction, anxiety, and depression. ${ }^{32}$ There were no differences between children who perceived their episodes of loss-of-control eating as large or not, with the exception of body dissatisfaction (higher in the group who perceived their episodes as large). These results suggest that the loss of control criterion, rather than the large size criterion, may be more closely associated with psychopathology. However, sample sizes in this study were small, making comparisons of children with larger vs. smaller episodes inconclusive. Another limitation of this study was that although all children were above the 85th percentile for age, children with loss of control episodes were heavier than those without these episodes.

Subjective Bulimic Episodes. Because the difference between the two types of bulimic episodes, OBEs and SBEs, lies in the quantity of food consumed, the pathological correlates of these episodes in individuals with eating disorders have been examined in research seeking to clarify the best criteria by which to define binge eating. For example, if SBEs are found to be as clinically significant as OBEs, this would suggest that the amount of food consumed may be a relatively less important criterion than the experience of a loss of control. For example, greater pathological "dieting" (as measured by combining scores on the Eating Attitudes Test $^{33}$ Diet subscale and Oral Control subscale, and the Eating Disorder Inventory ${ }^{34}$ Drive for Thinness subscale) was associated with more frequent SBEs but not with more frequent OBEs among inpatients with a variety of eating disorders involving binge eating (BN, anorexia nervosa, or eating disorders not otherwise specified). ${ }^{35}$ These authors of this study suggested that attempts at strict dieting may lead to cognitive consequences, such as disinhibition or the perception that one has lost control over eating, rather than behavioral consequences, such as the intake of large amounts of food.

Evidence that the occurrence of SBEs may be as important a symptom as OBEs comes from studies of binge episodes in women with BED or BN. Median splits were made of the scores of 101 women in treatment for BED on the Binge Eating Scale, ${ }^{36}$ Beck Depression Inventory, ${ }^{37}$ Three Factor Eating Questionnaire, ${ }^{38}$ and the Symptom Checklist- $90,{ }^{39}$ and the SBE and OBE frequencies of high versus low scorers were compared. ${ }^{40}$ Neither binge type's frequency was related to psychopathology. Those who eventually responded well to treatment did not differ from those who did not on either binge 
frequency at week one. Similarly, differences in frequency between subjective and objective binges in participants with BN did not account for the variance on any measures of psychopathology, diagnosis, global functioning, or weight. ${ }^{41}$ Keel et al. ${ }^{42}$ found no differences between women with BN with frequent OBEs and those with only frequent SBEs in restraint, disinhibition, hunger, or general psychopathology, but women with OBEs had greater (1) frequency of binge/purge episodes, and (2) impulsiveness (as measured by the Barratt Impulsiveness Scale-11; Ref. 43). In a community sample, women were examined who did not have an eating disorder, or had BED, BN, or subthreshold variants of these disorders (symptoms that fell short of meeting criteria for BED or BN but were greater than having no eating disorder, such as two OBEs, three SBEs, and one episode of self-induced vomiting in the past month). ${ }^{44}$ Comparable and significant associations were found between frequency of SBEs and OBEs with eating disorder psychopathology (including all four EDE subscales) and general psychopathology (such as depressed mood and anxiety). (The EDE subscales include Restraint, Eating Concern, Shape Concern, and Weight Concern; these subscales assess the core dysfunctional attitudes and behaviors that are often present in eating disorders. $)^{9}$

There is little research on the association between impairment in quality of life and different forms of binge eating, but recent data in a community sample of 5,232 women found that SBEs were associated with significant functional impairment and eating disorder psychopathology. SBEs were particularly associated with dysfunction when present in combination with compensatory behaviors such as self-induced vomiting and laxative abuse. ${ }^{45}$ Similarly, in a community sample of 214 women, Participants who experienced SBEs at least twice per month showed impairment in quality of life, particularly in the mental health domain, relative to participants who did not have SBEs. (This greater impairment was also found for participants with OBEs relative to those without OBEs.) However, there were no differences in impairment between participants who had twice-monthly objective overeating episodes (without a loss of control) relative to those who did not have objective overeating episodes. ${ }^{46}$ These results suggest that the regular experience of a loss of control over eating may impair quality of life, while the experience of overeating without a loss of control does not.

Although OBEs are nearly always used as a major outcome measure of the effects of treatment for $\mathrm{BED}$ or $\mathrm{BN}$, there is evidence that SBEs may be more unrelenting. Research has documented that response to cognitive-behavioral therapy (CBT) for eating disorders can often occur early in treatment, after initial behavioral interventions have been introduced. ${ }^{47}$ However, despite the early decrease in OBE frequency in response to CBT for BED, SBEs are slower to decrease $\mathrm{e}^{40}$ and may often persist after treatment. ${ }^{48}$ Hildebrandt and Latner ${ }^{49}$ examined a minimal behavioral intervention with women with BED or BN, involving a week of self-monitoring of food intake. Self-monitoring is the first intervention prescribed in CBT for BED and $\mathrm{BN}^{50}$ and may account for the early response to $\mathrm{CBT}^{47}{ }^{4}$ Indeed, an investigation of the effects of self-monitoring showed a significant decrease in OBE frequency from baseline to the self-monitoring phase in women with BED or BN. ${ }^{51}$ However, although OBE frequency decreased in response to a period of selfmonitoring, SBE frequency increased during selfmonitoring (a pattern termed "binge drift"; Ref. 49). These results suggest that this type of binge episode may prove more tenacious and difficult to treat. It is possible that two different sets of antecedents function to increase the probability of the two aspects of binge episodes. Excessive food intake may be more likely to result from negative energy balance or increased energy needs. On the other hand, a loss of control may result from negative affect or failed attempts to follow strict dietary rules. Thus, behavioral techniques used early in CBT might target the amounts of food consumed, whereas later cognitive techniques might be necessary to address patients' perceived loss of control.

A limitation of studies assessing SBEs using the $\mathrm{EDE}^{9}$ is their poor test-retest reliability, documented in multiple studies (e.g., Refs. 52 and 53). However, differences in SBEs over time may reflect natural fluctuations in the behavior over time, such as "binge drift." 49 The consensus of studies suggesting a relationship between SBEs and eating pathology is difficult to reconcile with the poor reliability of their assessment, but future research should develop more precise and sophisticated assessment tools for measuring the experience of loss of control.

Summary: Adults and children who experience a loss of control over eating exhibit greater psychopathology, relative to overeaters who do not lose control or to normal eaters. The loss of control over eating may be a primary criterion by which binge eaters define their own binge episodes, though its perception may be affected by mood. Overall, research on SBEs suggests the experience of a loss of control over eating may be a clinically significant and meaningful symptom (even when control is 
lost while eating only a small or moderate amount of food), and it may be relatively resistant to treatment. This symptom is associated with eatingrelated and general psychopathology, and it may require specific attention in treatment.

\section{Criteria for Binge Episodes in BED}

\section{Frequency of Episodes}

While some studies have shown that greater frequency of binge eating can signal more severe psychopathology, other investigations have shown similar psychopathology between higher versus lower frequency binge eaters. Some of these studies used treatment seeking samples, which may differ from nontreatment seeking populations in the presentation of associated eating pathology. Furthermore, the use of nonstandardized interviews or self-report measures to establish diagnoses may limit the conclusions drawn from several of these investigations. Comparisons across studies are difficult because of differing methodologies.

In a population-based survey, individuals with full-syndrome BED reported greater sadness, lower self-esteem, and more stress than individuals with a subthreshold variant of BED, defined as having a minimum of only one episode of binge eating per week. ${ }^{29}$ These low-frequency binge eaters in turn had more sadness and lower self-esteem than controls, suggesting a continuum of severity. In a community sample of 50 individuals with BED, greater frequency of binge eating episodes was correlated with higher body mass index (BMI, $\mathrm{kg} / \mathrm{m}^{2}$ ) and greater prevalence of personality disorders. ${ }^{54}$ (Binge size was not related to psychopathology, but only to BMI.) The findings suggest that binge frequency may signal the presence of greater comorbid psychopathology. In contrast, other research has shown similar impairments in general functioning between full BED and partial BED. ${ }^{55}$ (Partial BED was defined as meeting all SCID criteria for BED except binge frequency, which occurred at least 1 day per month for the last 6 months.) Frequency of binge eating did not affect levels of selfesteem, social adjustment, and past and current depression.

Research has also examined the differences in eating-related psychopathology in individuals with higher vs. lower frequencies of binge eating, and results have again been mixed. Individuals diagnosed with full BED reported earlier onset of binge eating, increased food cravings, increased diet pill use, a greater fear of weight gain, and increased body perception disturbance relative to those with partial BED (i.e., those who had recurrent binge eating but at a significantly less frequent rate than full BED). ${ }^{56}$ Fitzgibbon et al. ${ }^{57}$ found that those with full BED had greater drive for thinness and less interoceptive awareness than those with partial BED, defined as meeting all the criteria for BED except for frequency (any endorsement of binge eating in the last 6 months met criteria for partial $\mathrm{BED})$. However, these two investigations did not use a standardized clinical interview to establish diagnoses. ${ }^{58}$

On the other hand, Striegel-Moore et al. ${ }^{29}$ found that full-syndrome BED did not differ from partialBED (having a minimum of one binge episode per week) on dieting, weight history, and body image disturbance. However, after controlling for differences in BMI, the full BED group reported attaching greater importance to their weight. This study was limited by the lack of investigator-based assessment of both diagnoses and concurrent eating pathology. In a subsequent replication of this study using a structured diagnostic interview, the EDE, ${ }^{9}$ to establish diagnoses, Striegel-Moore et al. ${ }^{59} \mathrm{com}$ pared three groups of age-matched and ethnicitymatched community women: those with full BED, subthreshold BED, and healthy controls. Women who met all the criteria for BED except the binge frequency criterion (and who binged no less than once per month) were diagnosed with subthreshold BED. Before controlling for BMI, women with subthreshold BED scored significantly lower on shape concern than those with full BED. After controlling for BMI, these differences no longer remained and both groups scored similarly on shape concern. No significant differences were found on the restraint and weight concern subscales, before and after controlling for BMI. Similarly, with the exception of shape concern subscale of the EDE, on which 104 individuals with full syndrome BED scored higher than 45 individuals with partial BED (binge episodes at least 1 day per month for the past 6 months), those with full BED did not differ from partial BED on any other measures of eating pathology. $^{55}$

Summary: Thus, research using diverse samples and methods has provided some support for the hypothesis that greater binge frequency, or meeting the full frequency criterion for the BED diagnosis, is associated with greater eating-related and general psychopathology. This would suggest that the current DSM-IV frequency criterion is appropriate. At the same time, however, other studies provide evidence for the contrasting hypothesis that less 
frequent binge eaters, who do not meet the DSMIV criterion, are equally at risk of eating-related and general psychopathology to those with full BED.

\section{Additional Criteria}

The DSM stipulates five additional criteria associated with binge eating episodes in BED. For a diagnosis of BED to be met, an individual's binge episodes must be associated with three or more of the following: eating much more rapidly than normal, eating until feeling uncomfortably full, eating large amounts of food when not hungry, eating alone because of embarrassment over how much one is eating, and feeling disgust, guilt, or depression after overeating. Only limited research has examined the appropriateness of these criteria. Interestingly, there is a degree of overlap, or repetition, across the different criteria of BED. The criterion that binge episodes involve eating rapidly is closely related to the criterion that binge episodes need to occur within a discrete period of time. The criterion that overeating is accompanied by feeling disgust, guilt, or depression overlaps with the required criterion that distress over binge eating is present. Research on both of these overlapping sets of criteria is limited, especially for the former criteria; therefore, these will be discussed together in the following sections.

Eating Rapidly and in a Discrete Period of Time. The research of Johnson et al. ${ }^{24,25}$ has supported the criterion that binges must occur within a short period of time. The actors in the condition that was judged most binge-like had eaten large quantities of food in a brief period (nine cookies in $3 \mathrm{~min}$ ); these findings support the criterion of eating more rapidly than normal. In addition, individuals with BED have been found to eat at a substantially faster rate during binge episodes than during nonbinge episodes. $^{22}$

Eating until Uncomfortably Full. Research on the gastric capacity of individuals with BED and BN has used participants' estimates of abdominal discomfort as a measure of this capacity. Women with BN and obese women with frequent binge eating had greater gastric capacity than healthy women or nonbinge eating obese women, indicating more tolerance of fullness and less abdominal discomfort, rather than more discomfort, relative to control participants. ${ }^{26,27}$ Mitchell et al. ${ }^{60}$ also found less physical discomfort following binge episodes in BED than in BN, suggesting that the BED criterion of feeling uncomfortably full after eating might be reconsidered. Like the negative affect criterion (discussed below), this criterion might better classify individuals with $\mathrm{BN}$ than individuals with BED.
By interrupting participants after each 75 grams of food they consumed and having them complete visual analogue scales, changes in subjective experiences over the course of binge eating episodes were measured. ${ }^{61}$ This study demonstrated that even some healthy women report increasing feelings of sickness during binge episodes. Further research of this type is needed to identify the appropriateness of the criterion of feelings of discomfort at the end of binge episodes.

Eating When Not Hungry. The criterion that binge episodes are associated with eating large amounts of food when not hungry is supported by literature on the appetite deficiencies in individuals with frequent binge eating. However, much of this research suggests disturbances in the termination of eating, rather than in its initiation, ${ }^{10}$ and the majority of this research has been conducted on binge eating in $\mathrm{BN}$. Over the course of eating episodes, women with $\mathrm{BN}$ report little increase in fullness relative to control participants. ${ }^{62}$ They also report less fullness at the end of meals ${ }^{63,64}$; and less decrease in their desire for foods that have been recently eaten (sensory specific satiety ${ }^{65}$ than controls). Satiety-related physiological disturbances that are associated with binge eating, in cholecystokinin, ${ }^{66}$ ghrelin, ${ }^{67}$ peptide $\mathrm{YY}^{68}$ and gastric distention, ${ }^{26}$ may account for patients' difficulties with terminating eating. Further investigation is needed to examine potential disturbances in BED. Nonetheless, perhaps this criterion could be phrased in a way that better reflects the research findings on difficulty terminating (rather than initiating) eating (e.g., "continuing to eat large amounts of food when no longer hungry").

Embarrassment and Eating Alone. It is widespread clinical knowledge that most binge episodes occur in private, and often in secret. This is commonly linked to the shame and embarrassment associated with binge eating, and limited research supports this link. For example, shame was associated with greater bulimic symptoms in both undergraduate women and women with $\mathrm{BN} .{ }^{69}$ Women who binge eat also have greater fluctuations in feelings of shame and guilt, as well as other negative affect, than women who do not binge eat. However, levels of shame and guilt did not rise prior to binge eating; instead, self-esteem and positive affect increased before binge episodes. ${ }^{70}$ A qualitative study of 13 women with BN analyzed interviews about their experiences and found the most prominent theme to be isolating the self, which included such behaviors as concealing eating and sneaking food. ${ }^{71}$ A limitation of these studies is that they did not measure embarrassment and eating alone 
specifically. Research is needed to identify the clinical significance of this criterion in BED.

Negative Affect after Overeating and Distress Over Binge Eating. The association between BED and general psychopathology, ${ }^{29}$ such as co-morbid depression, may shed some light on the validity of the criterion of feeling disgusted with oneself, depressed, or guilty about overeating, as well as on the criterion that marked distress regarding binge eating must be present. Functional analyses of eating disorders and binge eating have also provided nuanced information about the immediate and delayed consequences of problem eating behaviors (see Ref. 72 for a review). Research with college women who self-report frequent binge eating has shown that negative affect is relieved by binge eating, at least in the short-term. ${ }^{73}$ Although the positive effect of this negative reinforcement may be only temporary, the impact of binge eating on mood may be significantly less negative for BED than for BN. In $\mathrm{BED}$, significantly less anxiety and distress have been shown to follow binge episodes than in BN. ${ }^{60}$ Again, it may seem surprising that this criterion exists only for BED, when it may better describe the experience of individuals with BN.

Summary: Existing research provides moderate support for the additional five criteria required for the diagnosis of BED. Binge eating may involve eating rapidly and in a short time-frame, and eating until uncomfortably full. It also seems that uncomfortable fullness may follow overeating or gastric distension among individuals without BED as well. It is clearer that binge eating involves persisting in eating when no longer hungry, but research is needed to examine hunger levels of binge eaters before binge episodes are initiated. While only limited research and clinical experience support the criterion of embarrassment and eating alone, more evidence suggests a link between binge eating and general distress or negative mood. However, the sequence of binge eating and negative affect requires further study; negative affect may precede and trigger bingeing, followed by short-term relief and positive affect that may ultimately give way again to longer-term distress. Finally, the overlap in criteria regarding speed/time of eating and distress regarding overeating and might be reconsidered, as there may be a more focused and concise method of capturing these constructs.

\section{Distinguishing BED from BN}

Do the criteria needed to meet the diagnosis of BED have discriminant validity? The independence and integrity of the criteria that comprise it depend largely on whether BED can be distinguished from other disorders that share similar characteristics, ${ }^{74}$ or "neighboring syndromes." 7 Two of the major qualitative distinctions between the diagnostic criteria of BED and BN are compensatory behaviors and shape and weight concerns. Purging or other compensatory behaviors are required for a diagnosis of $\mathrm{BN}$, while these behaviors are exclusionary criteria for (and thus absent in) BED. ${ }^{55,58,75,76}$ In $\mathrm{BN}$, self-evaluation must be unduly influenced by body shape and weight, but no such criterion exists for BED. ${ }^{1,77}$ Differences in weight and weight history such as greater weight cycling ${ }^{78}$ and higher BMI and rates of obesity in BED ${ }^{17,29}$ may be related to the absence of compensatory behaviors. An examination of the differences in psychopathology between BED and BN can reflect on whether BED's lack of a diagnostic criterion relating to shape and weight overconcern are appropriate.

Studies comparing the eating disorder psychopathology associated with $\mathrm{BN}$ to that associated with BED have typically found that BN is associated with more severe eating pathology. For example, patients with BN were higher not only on measures of purging, but also on avoidance of forbidden foods, restrictive eating, fear of fatness, ${ }^{75}$ restraint, and disinhibition ${ }^{55}$ than those with BED. Despite greater EDE restraint and eating concerns in $\mathrm{BN}$ compared to $\mathrm{BED}$, Wilfley et al. ${ }^{79}$ documented more secretive eating in BED participants. Obese BED patients reported significantly lower drive for thinness than BN and nonobese BED patients, who did not differ from each other. However, body dissatisfaction did not differ between these groups. ${ }^{80}$ BED closely resembled $\mathrm{BN}^{55}$ on the shape concern and weight concern subscales of the questionnaire version of the EDE (EDE-Q), ${ }^{16}$ even when controlling for the effect of BMI. ${ }^{17}$ Studies controlling for BMI are especially conclusive since individuals with BN are often not overweight (e.g., Refs. 28 and 81).

Thus, despite higher levels of eating disorder psychopathology and dietary restraint in BN (which may be related to the presence or absence of compensatory behaviors), similarities in shape and weight concerns between BN and BED have been reliably documented. ${ }^{55,75,78,82,83}$ This suggests that weight and shape overconcern may be sufficiently elevated to be considered as a diagnostic criterion for BED as it is for BN. Even though BED does not currently have a cognitive criterion concerning shape and weight concern, future versions of the DSM (e.g., DSM-V) could consider stipulating that self-evaluation is unduly influenced by body shape 
and weight in BED. The inconsistency between the DSM shape and weight concern criteria for BN and BED are surprising given the similarity in psychopathology between them. A more comprehensive set of criteria that includes shape and weight concern might more accurately capture the core psychopathology of individuals with BED.

Evidence is mixed on whether BN and BED differ in associated general psychopathology. Several studies comparing BN and BED have demonstrated significantly greater levels of depression, ${ }^{84,85}$ anxiety, ${ }^{85,86}$ personality disorders, ${ }^{85}$ social adjustment, ${ }^{87}$ general distress, impairment, and comorbid psychiatric symptoms ${ }^{84,87}$ in BN. However, other studies have documented no significant differences between BN and BED in levels of state or trait depression, ${ }^{55,75,78,80,86}$ feelings of sadness ${ }^{78}$ self-esteem or social adjustment, ${ }^{55}$ suicide attempts, ${ }^{75}$ self-injurious behavior or drug abuse, ${ }^{75,78,82}$ history of hospitalization, ${ }^{86}$ and other comorbid psychopathology. ${ }^{75,86,88}$ Some of these studies are limited by methodological concerns such as self-report measures for establishing diagnoses, use of treatment-seeking samples, and small samples resulting in a lack of statistical power. Comparisons between studies are often difficult as researchers have frequently used different instruments to measure areas of psychopathology and functioning.

Summary: Research has demonstrated that although BED is associated with lower eatingrelated psychopathology than $\mathrm{BN}$, their body dissatisfaction and shape and weight concerns are similarly high. Evidence is mixed regarding general psychopathology, with some studies showing higher levels among BN than BED, and other studies demonstrating similar levels of impairment between the groups.

\section{Distinguishing BED from Obesity without Binge Eating}

Because BED is often accompanied by obesity, it is valuable to consider the distinction between individuals with obesity with BED and individuals with obesity without BED. These distinctions reflect on the question of whether obesity should be included as a diagnostic criterion for BED. If the distress and psychopathology associated with BED are largely or primarily due to accompanying obesity, then obesity should be considered for inclusion as a diagnostic criterion. Research thus far suggests that this is not the case. The differences between BED and obese individuals without binge eating fall into the broad categories of eating pathology and eating patterns, general psychopathology, and quality of life.

Obese individuals with BED have been shown to have more severe eating pathology than obese individuals without $\mathrm{BED}$. They have greater concerns about shape and weight, with more body dissatisfaction than similarly obese individuals without frequent binge eating. ${ }^{5}$ While the shape and weight concerns of individuals with BED resemble those of individuals with $\mathrm{BN}$ or anorexia nervosa (as discussed above) [e.g., Ref. 17], the body dissatisfaction of obese patients with BED is greater than that of non-BED obese patients. ${ }^{80}$ In addition to the presence of frequent binge eating, the eating patterns and dietary composition of individuals with obesity and BED differ from individuals with obesity without BED, as discussed above. ${ }^{11,13}$

Larger binge episode size has been demonstrated with increased obesity in BED, ${ }^{11}$ and greater adiposity has been linked with more severe binge eating. ${ }^{89}$ However, the psychological distress associated with binge eating may be more closely associated with binge eating severity than with the degree of obesity. For example, greater binge severity on the Binge Eating Scale ${ }^{36}$ was related to greater depression, psychological distress, poorer self-esteem, and interpersonal problems. ${ }^{20}$ The overall lack of differences between those women with BED who do and do not have concurrent obesity, on eating related psychopathology, general personality, and depression, strongly suggests that the problems associated with BED are unrelated to the presence or degree of comorbid obesity. ${ }^{80}$ Didie and Fitzgibbon ${ }^{90}$ compared women diagnosed with BED from across three weight categories: normal/overweight, obese, and severely obese. There were no differences between the groups in general psychiatric distress, depressive symptoms, or eating disturbances. These studies suggest that binge eating, rather than excess weight, may drive the distress and eating disturbances present in obese binge eaters. The evidence also indicates greater psychiatric disturbance in obese individuals with BED than in obese individuals without BED. Treatment-seeking samples and community-based samples of BED both show increased rates of psychiatric disturbance relative to obese non-BED samples. ${ }^{5}$

An investigation comparing 177 individuals with BED to 45 overweight individuals found several essential differences were found between these groups. ${ }^{91}$ Symptoms of depression were significantly higher among individuals with BED, as were eating-related psychopathology, including EDEassessed OBEs, dietary restraint, eating concerns, 
disinhibition, and hunger. Despite the similar BMI of the groups, weight concern and shape concern were significantly more disturbed in those with BED. They also ate more frequently during the day than either the overweight comparison participants or individuals with night eating syndrome. This study provided definitive evidence for the distinctiveness of BED from obesity without BED.

Despite the differences between obesity with BED and obesity without BED, it is possible that the groups may have similar responses to obesity treatment, at least in the short term. In both groups, weight losses following behavioral weight control treatment have been shown to be similar, with reductions in binge eating in the BED group. ${ }^{92}$ This similarity may also exist in self-help treatment, where frequent binge eaters lost as much weight as nonbinge-eaters after 1 year of treatment. ${ }^{93}$ However, these studies used self-report questionnaires to diagnose binge eating. There is also some evidence of greater attrition from weight loss treatment among obese individuals with $\mathrm{BED},{ }^{94}$ and studies with longer follow-up periods are needed to examine the long-term effects of weight loss on binge eating.

Yet the increased psychiatric problems, eating pathology, and medical comorbidity in BED, even independent of obesity, ${ }^{95,96}$ may take a significant toll on the quality of life of individuals with BED. Rieger et al. ${ }^{3}$ investigated the scope of impairment associated with BED in treatment-seeking obese individuals, relative to obese individuals without BED. Individuals with BED were more impaired on overall quality of life and in the domains of work, public distress, sexual life, and self-esteem. The groups were similar in weight. Therefore, the differences between them appeared to be attributable to the presence of BED, which may be associated with decreased quality of life even beyond the impairment already caused by obesity.

Summary: Individuals with BED differ from those without BED in numerous clinically significant ways, even when both groups are overweight or obese. BED is associated with greater eating pathology, body image disturbance, and general psychopathology. They also report poorer health-related quality of life. The impairments associated with binge eating appear to be independent of weight level or obesity status.

\section{Conclusions and Clinical Implications}

The DSM-IV-TR definition of a mental disorder includes present distress, disability, or significantly increased risk of suffering. ${ }^{1}$ Tanofsky-Kraff and Yanovski, ${ }^{31,97}$ in their recommendations for refining the diagnostic criteria for eating disorders that may occur in obese individuals, have suggested that "to assess the clinical significance of these mental disorders adequately, criteria should ideally be based on variables that relate to distress and functional impairment associated with the nonnormative eating" (p. 1364). These authors suggested that eating behaviors that are non-normative be classified as disordered eating only if they cause distress or impairment. Examples given by these authors of non-normative eating that may contribute to energy balance, but are not necessarily impairing or distressing, included objective overeating episodes without a loss of control, irregular meal patterns, or frequent snacking.

Research findings are mixed on whether the 2day-per-week criterion is the appropriate cut-off point for average binge episode frequency. Some investigations have demonstrated significant psychopathology in individuals who binge eat at a less frequent rate. Some research also supports the criterion of a large amount of food (e.g., Ref. 14). However, several studies suggest that the experience of loss of control over eating, even while consuming small or moderate amounts of food, may be at least as closely linked with eating-related and general psychopathology as is the experience of binge eating on large amounts of food (e.g., Refs. 40 and 42). It may be that the symptom most associated with distress and functional impairment is loss-of-control eating, and that this should be the key variable in defining binge eating. Patients with restrictive dietary patterns may have especially low thresholds for breaking their restrictions. In these individuals, disinhibition or loss of control, rather than the quantity of food consumed, may more accurately characterize and capture binge episodes and the pathology with which they are associated. Overall, studies that have examined the key defining components of binge eating episodes have often pointed to the loss of control as a criterion of primary importance. Therefore, future definitions of BED should consider including as binges those episodes involving a loss of control over eating while consuming small or moderate amounts of food.

Only limited evidence has provided support for the inclusion of the additional criteria associated with binge episodes in BED. Although not all of these criteria are required to qualify for a diagnosis of BED, further research is needed to clarify whether these criteria aptly characterize BED. Ironically, more research has supported some of 
TABLE 1. Key conclusions of research evidence addressing diagnostic criteria for BED

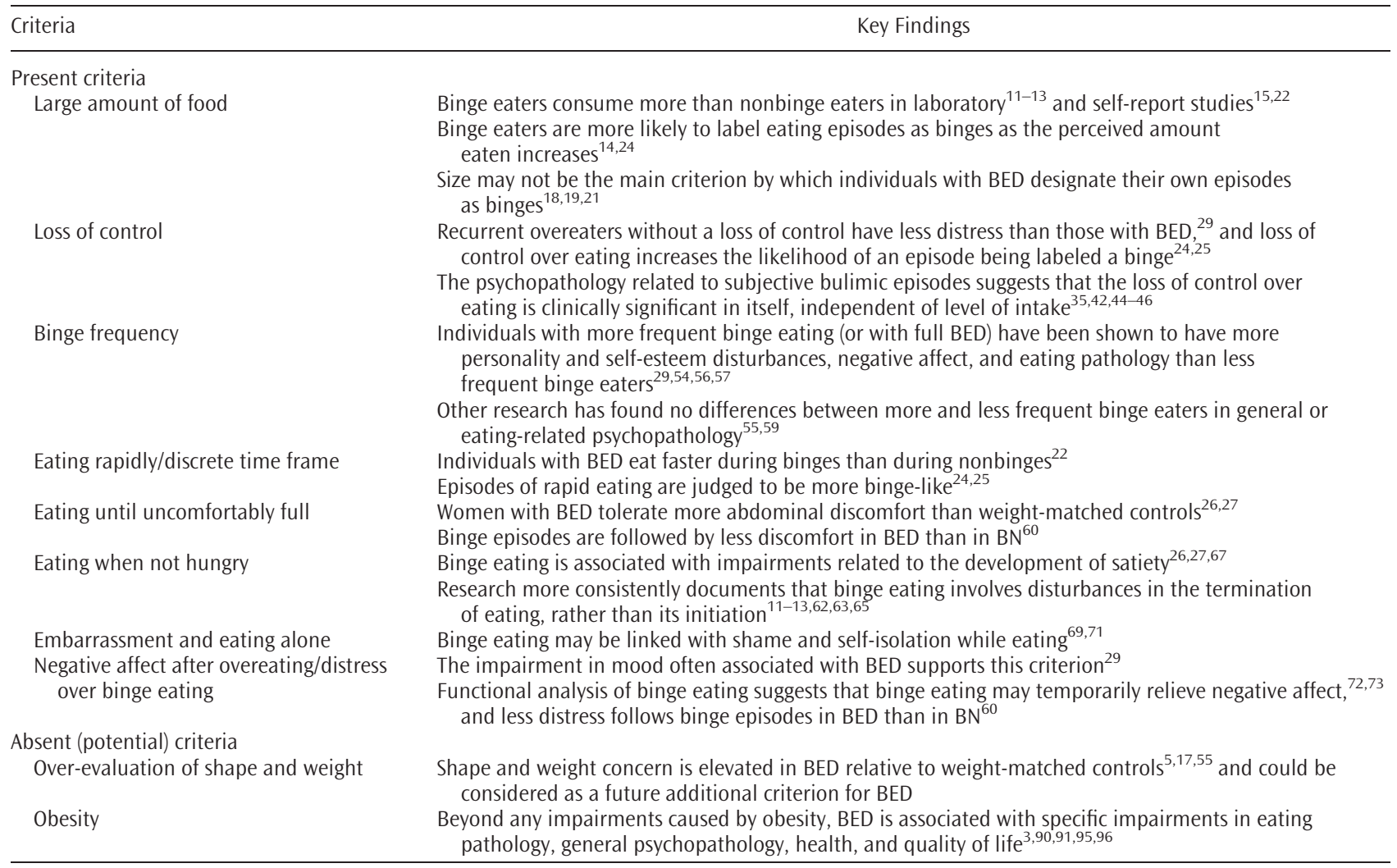

these associated features of binge episodes in individuals with $\mathrm{BN}$, where these criteria are not required. To our knowledge, no published research has been conducted on individuals who meet three out of these five criteria when compared to individuals who do not meet three out of the five criteria. Such research is needed to determine the validity of these criteria.

Research examining the differences between BED and BN suggests that although these disorders may often differ on general psychopathology and eating-related psychopathology, both groups experience significant shape and weight concerns. Shape and weight overevaluation is a criterion for BN but not for BED, yet it fails to discriminate between these disorders. Indeed, recent research has suggested that individuals with BED who place exaggerated importance on shape and weight in their self-evaluation have significantly greater eating-related and general psychopathology, and poorer quality of life, than individuals with BED who do not overvalue shape or weight. ${ }^{98,99}$ Therefore, this criterion might be considered as a potential addition to future definitions of BED. On the other hand, the consensus of research suggests that an additional criterion of obesity or overweight would probably not add any valuable information to the current set of criteria, as the problems associated with BED appear more closely related to the presence of binge eating than to co-occurring obesity. Table 1 highlights the key findings that can be concluded about the diagnostic criteria for BED based on existing evidence.

Future research should compare the psychopathology and functioning between individuals with full and partial BED, where partial BED defined as those with subthreshold binge size criterion (SBEs rather than OBEs). Such research would be valuable in determining the diagnostic validity of the size criterion for defining binge episodes in BED. Additional methodological advances in future research could also improve on the studies reviewed here. The studies reviewed here primarily examined eating syndromes cross-sectionally. However, BED has a variable course ${ }^{100,101}$ Longitudinal research comparing the course and outcome in those that meet full criteria for BED with the course of eating pathology in those that fall short on one or more criteria could provide useful information about the clinical utility of the current diagnostic criteria. A methodological concern of many studies is the sole use of treatment-seeking 
populations, limiting the generalizability of results. More community-based studies are needed.

These conclusions have important implications for clinical practice and the provision of treatment to people in need. The evidence presented in this review suggests that binge eating problems which do not meet full criteria for BED, because of factors such as insufficient frequency or size of episodes, can often cause intense distress and impairment for the individuals who experience them. Individuals with these forms of binge eating problems are often in great need of treatment and can benefit from it. It is possible that stepped-care treatment approaches, ${ }^{102}$ which begin with pure self-help, guided self-help, ${ }^{103}$ or psychoeducational treatment, ${ }^{104}$ might help to pinpoint a subset of milder or subthreshold cases that respond to more minimal interventions. However, much more research is needed to evaluate the relative effectiveness of treatment and self-help treatment with partial versus full-syndrome BED. Future treatment studies should include broader samples of both full- and partial-syndrome patients, in order to examine diagnostic issues and differences in prognosis, within a treatment context.

We acknowledge Juliet Rosewall for her helpful assistance, and two anonymous reviewers for their helpful comments on an earlier version of this manuscript.

\section{References}

1. American Psychiatric Association. Diagnostic and Statistical Manual of Mental Disorders (Text Revision), 4 ed. Washington, DC: American Psychiatric Press, 2000.

2. Johnson JG, Spitzer RL, Williams BW. Health problems, impairment and illnesses associated with bulimia nervosa and binge eating disorder among primary care and obstetric gynaecology patients. Psychol Med 2001;31:1455-1466.

3. Rieger E, Wilfley DE, Stein RI, Marino V, Crow SJ. A comparison of quality of life in obese individuals with and without binge eating disorder. Int J Eat Disord 2005;37:234-240.

4. deZwaan M, Mitchell JE, Howell L, Monson N, Swan-Kremeier L, Roerig JL, et al. Two measures of health-related quality of life in morbid obesity. Obes Res 2002;10:1143-1151.

5. Wilfley DE, Wilson GT, Agras WS. The clinical significance of binge eating disorder. Int J Eat Disord 2003;34(Suppl):S96-S106.

6. Stunkard AJ, Allison KC. Binge eating disorder: Disorder or marker? Int J Eat Disord 2003;34(Suppl):S107-S116.

7. Kendell R, Jablensky A. Distinguishing Between the validity and utility of psychiatric diagnoses. Am J Psychiatry 2003; 160:4-12.

8. Cooper Z, Fairburn CG. Refining the definition of binge eating disorder and nonpurging bulimia nervosa. Int J Eat Disord 2003;34(Suppl):S89-S95.

9. Fairburn CG, Cooper Z. The eating disorder examination. In: Fairburn CG, Wilson GT, editors. Binge Eating: Nature, Assessment, and Treatment, 12th ed. New York: Guilford Press, 1993;317-332
10. Walsh BT, Boudreau G. Laboratory studies of binge eating disorder. Int J Eat Disord 2003;34(Suppl):S30-S38.

11. Guss JL, Kissileff HR, Devlin MJ, Zimmerli E, Walsh BT. Binge size increases with body mass index in subjects with binge eating disorder. Obes Res 2002;10:1021-1029.

12. Raymond NC, Bartholome LT, Lee SS, Peterson RE, Raatz SK. A comparison of energy intake and food selection during laboratory binge eating episodes in obese women with and without a binge eating disorder diagnosis. Int J Eat Disord 2007; 40:67-71.

13. Yanovski SZ, Leet M, Yanovski JA, Flood M, Gold PW, Kissileff $H R$, et al. Food selection and intake of obese women with and without binge eating disorders. Am J Clin Nutr 1992;56: 979-980.

14. Guertin TL, Conger AJ. Mood and forbidden foods' influence on perceptions of binge eating. Addict Behav 1999;24:175193.

15. Greeno CG, Wing RR, Marcus MD. How many donuts in a "binge"? Women with BED eat more but do not have more restrictive standards than weight-matched non-BED women. Addict Behav 1999;24:299-303.

16. Fairburn CG, Beglin S. Assessment of eating disorders: Interview or self-report questionnaire? Int J Eat Disord 1994;16: 363-370.

17. Masheb RM, Grilo CM. Binge eating disorder; a need for additional diagnostic criteria. Compr Psychiatry 2000;41:159-62.

18. Rosen JC, Leitenberg H, Fisher C, Khazam C. Binge-eating episodes in bulimia nervosa: The amount and type of food consumed. Int J Eat Disord 1986;5:255-267.

19. Telch CF, Pratt EM, Niego SH. Obese women with binge eating disorder define the term binge. Int J Eat Disord 1998;24:313317.

20. Telch CF, Agras WS. Do emotional states influence binge eating in the obese? Int J Eat Disord 1996;20:271-279.

21. Raymond NC, Neumeyer B, Warren CS, Lee SS, Peterson CB. Energy intake patterns in obese women with binge eating disorder. Obes Res 2003;11:869-879.

22. Rossiter EM, Agras WS, Telch CF, Bruce B. The eating patterns of non-purging bulimic subjects. Int J Eat Disord 1992;11: 111-120.

23. Spitzer R, Williams JB, Gibbon M, First MB. The structured clinical interview for DSM-III-R. Arch Gen Psychiatry 1992; 49:624-629.

24. Johnson WG, Robertson-Nay R, Rohan KJ, Torgrud L. An experimental investigation of DSM-IV binge-eating criteria. Eat Behav 2003;4:295-303.

25. Johnson WG, Boutelle KN, Torgrud L, Davig JP, Turner S. What is a binge? The influence of amount, duration, and loss of control criteria on judgments of binge eating. Int J Eat Disord 2000;27:471-479.

26. Geliebter A, Hashim SA. Gastric capacity in normal, obese, and bulimic women. Physiol Behav 2001;74:743-746.

27. Geliebter A, Melton PM, McCray RS, Gallagher DR, Gage D, Hashim SA. Gastric capacity, gastric emptying, and test-meal intake in normal and bulimic women. Am J Clin Nutr 1992; 56:656-661.

28. Geliebter A. New developments in binge eating disorder and the night eating syndrome. Appetite 2002;39:175-177.

29. Striegel-Moore RH, Wilson GT, Wilfley DE, Elder KA, Brownell KD. Binge eating in an obese community sample. Int J Eat Disord 1998;23:27-37

30. Thelen $\mathrm{MH}$, Farmer J, Wonderlich S, Smith M. A revision of the Bulimia test: The BULIT-R. Psychol Assess 1991;3:119-124.

31. Tanofsky-Kraff M, Yanovski SZ, Wilfley DE, Marmarosh C, Morgan CM, Yanovski JA. Eating-disordered behaviors, body fat, and psychopathology in overweight and normal-weight children. J Consult Clin Psychol 2004;72:53-61. 
32. Morgan CM, Yanovski SZ, Nguyen TT, McDuffie NGS, Jorge MR, Keil M, et al. Loss of control over eating, adiposity, and psychopathology in overweight children. Int J Eat Disord 2002; 31:430-441.

33. Garner DM, Olmstead MP, Bohr Y, Garfinkel PE. The eating attitudes test: Psychometric features and clinical correlates. Psychol Med 1982;12:871-878.

34. Garner DM, Olmstead MA, Polivy J. Development of a multidimensional eating disorder inventory for anorexia nervosa and bulimia. Int J Eat Disord 1983;2:15-34.

35. Kerzhnerman I, Lowe MR. Correlates of subjective and objective binge eating in binge-purge syndromes. Int J Eat Disord 2002;31:220-228.

36. Gormally J, Black S, Daston S, Rardin D. The assessment of binge-eating severity among obese persons. Addict Behav 1982;7:47-55.

37. Beck AT, Steer RA, Ball R, Ranieri WF. Comparison of Beck depression inventories-IA and -II in psychiatric outpatients. J Pers Assess 1996;67:588-597.

38. Stunkard AJ, Messick S. The Three-factor Eating Questionnaire to measure dietary restraint, disinhibition and hunger. J Psychosom Res 1985;29:71-83.

39. Derogatis LR, Lipman R, Rickels K, Uhlenhath E, Covi L. The Hopkins symptoms check list ( $\mathrm{HSCL})$ : A self-report symptoms inventory. Behav Sci 1974;19:1-15

40. Niego SH, Pratt EM, Agras WS. Subjective or objective binge: Is the distinction valid? Int J Eat Disord 1997;22:291-298.

41. Pratt EM, Niego SH, Agras WS. Does the size of a binge matter? Int J Eat Disord 1998;24:307-312.

42. Keel PK, Mayer SA, Harnden-Fischer JH. Importance of size in defining binge eating episodes in bulimia nervosa. Int J Eat Disord 2001;29:294-301.

43. Patton J, Stanford MS, Barratt ES. Factor structure of the Barratt impulsiveness scale. J Clin Psychol 1995;51:768-774.

44. Latner JD, Hildebrandt T, Rosewall JK, Chisholm AM, Hayashi $\mathrm{K}$. Loss of control over eating reflects eating disturbances and general psychopathology. Behav Res Ther 2007;45:2203-2211.

45. Mond J, Hay P, Rodgers B, Owen C, Crosby R, Mitchell J. Use of extreme weight control behaviors with and without binge eating in a community sample: Implications for the classification of bulimic-type eating disorders. Int J Eat Disord 2006; 39:294-302.

46. Vallance J. The relationship between eating disorder psychopathology and quality of life within a non-clinical sample [masters' thesis]. Christchurch: University of Canterbury, 2006.

47. Wilson GT, Loeb KL, Walsh BT, Labouvie E, Petkova E, Liu X, et al. Psychological versus pharmacological treatments of bulimia nervosa: Predictors and processes of change. J Consult Clin Psychol 1999;67:451-459.

48. Walsh BT, Fairburn CG, Mickley D, Sysko R, Parides MK. Treatment of bulimia nervosa in a primary care setting. Am J Psychiatry 2004;161:556-561.

49. Hildebrandt T, Latner JD. Effect of self-monitoring on binge eating: Treatment response or "binge drift"? Eur Eat Disord Rev 2006;14:17-22.

50. Fairburn CG, Marcus MD, Wilson GT. Cognitive-behavioral therapy for binge eating and bulimia nervosa: A comprehensive manual. In: Fairburn CG, Wilson GT, editors. Binge Eating: Nature, Assessment, and Treatment. New York: Guilford Press, 1993, pp. 361-404.

51. Latner JD, Wilson GT. Self-monitoring and the assessment of binge eating. Behav Ther 2002;33:465-477 (Summary).

52. Grilo CM, Lozano C, Elder KA. Inter-rater and test-retest reliability of the spanish language version of the eating disorder examination interview: Clinical and research implications. J Psychiatr Pract 2005;11:231-240.
53. Grilo CM, Masheb RM, Lozano-Blanco C, Barry DT. Reliability of the eating disorder examination in patients with binge eating disorder. Int J Eat Disord 2004;35:80-85.

54. Picot AK, Lilenfeld LRR. The relationship among binge severity, personality psychopathology, and body mass index. Int J Eat Disord 2003;34:98-107.

55. Crow SJ, Agras WS, Halmi K, Mitchell JE, Kraemer HC. Full syndromal versus subthreshold anorexia nervosa, bulimia nervosa, and binge eating disorder: A multicenter study. Int J Eat Disord 2002;32:309-318.

56. Mussell MP, Mitchell JE, de Zwann M, Crosby RD, Seim HC, Crow SJ. Clinical characteristics associated with binge eating in obese females: A descriptive study. Int J Obes Relat Metab Disord 1996;20:324-331.

57. Fitzgibbon ML, Sanchez-Johnsen LAP, Martinovich Z. A test of the continuity perspective across bulimic and binge eating pathology. Int J Eat Disord 2003;34:83-97.

58. Wilfley DE, Friedman MA, Dounchis JZ, Stein RI, Welch RR, Ball SA. Comorbid psychopathology in binge eating disorder: Relation to eating disorder severity at baseline and following treatment. J Consult Clin Psychol 2000;68:641-649.

59. Striegel-Moore RH, Dohm FA, Solomon EE, Fairburn CC, Pike KM, Wilfley DE. Subthreshold binge eating disorder. Int J Eat Disord 2000;27:270-278.

60. Mitchell JE, Mussell MP, Peterson CB, Crow S, Wonderlich SA, Crosby RD, et al. The hedonics of binge eating in women with bulimia nervosa and binge eating disorder. Int J Eat Disord 1999;26:165-170.

61. Wentzlaff TM, Guss JL, Kisileff HR. Subjective ratings as a function of amount consumed: A preliminary report. Physiol Behav 1995;57:1209-1214.

62. Halmi KA, Sunday SR, Puglisi A, March P. Hunger and satiety in anorexia and bulimia nervosa. Ann NY Acad Sci 1989; 575:431-445.

63. Hadigan CM, Walsh BT, Devlin MJ, LaChaussee JL, Kissileff HR. Behavioral assessment of satiety in bulimia nervosa. Appetite 1992;18:233-241.

64. Geracioti TD, Liddle RA. Impaired cholecystokinin secretion in bulimia nervosa. N Engl J Med 1989;319:683-688.

65. Hetherington MM, Rolls BJ. Sensory-specific satiety in anorexia and bulimia nervosa. Ann NY Acad Sci 1989;575:387398.

66. Devlin MJ, Walsh BT, Guss JL, Kissileff HR, Liddle RA, Petkova E. Postprandial cholecystokinin release and gastric emptying in patients with bulimia nervosa. Am J Clin Nutr 1997;65: 114-120.

67. Geliebter A, Gluck ME, Hashim SA. Plasma ghrelin concentrations are lower in binge-eating disorder. J Nutr 2005;135: 1326-1330

68. Monteleone P, Martiadis V, Rigamonti AE, Fabrazzo M, Giordani $C$, Muller EE, et al. Investigation of peptide $Y Y$ and ghrelin responses to a test meal in bulimia nervosa. Biol Psychiatry 2005;57:926-931.

69. Hayaki J, Friedman MA, Brownell KD. Shame and severity of bulimic symptoms. Eat Behav 2002;3:73-83.

70. Sanftner JL, Crowther JH. Variability in self-esteem, moods, shame, and guilt in women who binge. Int J Eat Disord 1998; 23:391-397.

71. Broussard BB. Women's experiences of bulimia nervosa. J Adv Nurs 2005;49:43-50.

72. Farmer R, Latner JD. Eating disorders. In: Sturmey P, editor. Functional Analysis in Clinical Treatment. Boston, MA: Academic Press, 2007, pp. 379-402.

73. Deaver CM, Miltenberger RG, Smyth J, Meidinger A, Crosby R. An evaluation of affect and binge eating. Behav Modif 2003; 27:578-599. 
74. Devlin MJ, Goldfein JA, Dobrow I. What is this thing called BED? Current status of binge eating disorder nosology. Int J Eat Disord 2003;34(Suppl):S2-S18.

75. Martin CK, Williamson DA, Thaw JM. Criterion validity of the multiaxial assessment of eating disorders symptoms. Int J Eat Disord 2000;28:303-310.

76. Williamson DA, Gleaves DH, Stewart TM. Categorical versus dimensional models of eating disorders: An examination of the evidence. Int J Eat Disord 2005;37:1-10.

77. Becker A, Grinspoon S, Klibanski A, Herzog D. Current concepts: Eating disorders. N Eng J Med 1999;340:1092-1098.

78. Vervaet $\mathrm{M}$, van Heeringen $\mathrm{C}$, Audenaert K. Binge eating disorder and non-purging bulimia: More similar than different? Eur Eat Disord Rev 2004;12:27-33.

79. Wilfley DE, Schwartz MB, Spurrell EB, Fairburn CG. Using the eating disorder examination to identify the specific pathology of binge eating disorder. Int J Eat Disord 2000;27:259-269.

80. Barry DT, Grilo CM, Masheb RM. Comparison of obese patients with binge eating disorder and nonobese patients with binge eating disorder. J Nerv Ment Dis 2003;191:589-594.

81. Wilson GT, Fairburn CG. Treatments for eating disorders. In: Nathan PE, Gorman JM, editors. A guide to treatments that work, 2nd ed. New York: Oxford University Press, 2002;559592.

82. Dohm FA, Striegel-Moore RH, Wilfley DE, Pike KM, Hook J, Fairburn CG. Self-harm and substance use in a community sample of black and white women with binge eating disorder or bulimia nervosa. Int J Eat Disord 2002;32:389-400.

83. Santonastaso P, Ferrara S, Favaro A. Differences between binge eating disorder and nonpurging bulimia nervosa. Int J Eat Disord 1999;25:215-218.

84. Brody ML, Walsh T, Devlin MJ. Binge eating disorders; reliability and validity of a new diagnostic category. J Consult Clin Psychol 1994;62:381-386.

85. Raymond NC, Mussell MP, Mitchell JE, de Zwann M, Crosby RD. An age-matched comparison of subjects with binge eating disorder and bulimia nervosa. Int J Eat Disord 1995; 18:135-143.

86. Tobin DL, Griffing A, Griffing S. An examination of subtype criteria for bulimia. Int J Eat Disord 1997;22:179-186.

87. Hay P, Fairburn C. The validity of the DSM-IV scheme for classifying bulimic eating disorders. Int J Eat Disord 1998;23:7-15.

88. Striegel-Moore RH, Cachelin FM, Dohm FA, Pike KM, Wilfley DE, Fairburn CG. Comparison of binge eating disorder and bulimia nervosa in a community sample. Int J Eat Disord 2001;29:157-165.

89. Telch CF, Agras WS, Rossiter EM. Binge eating increases with increasing adiposity. Int J Eat Disord 1988;7:115-119.
90. Didie ER, Fitzgibbon M. Binge eating and psychological distress: Is the degree of obesity a factor? Eat Behav 2004;6: 35-41.

91. Allison KC, Grilo CM, Masheb RM, Stunkard AJ. Binge eating disorder and night eating syndrome: A comparative study of disordered eating. J Consult Clin Psychol 2005;73:11071115.

92. Gladis MM, Wadden TA, Vogt R, Foster G, Kuehnel RH, Bartlett $S J$. Behavioral treatment of obese binge eaters: Do they need different care? J Psychosom Res 1998;44:375-384.

93. Delinsky SS, Latner JD, Wilson GT. Binge eating and weight loss in a self-help behavior modification program. Obesity 2006;14:1244-1249.

94. Sherwood NE, Jeffrey RW, Wing RR. Binge status as a predictor of weight loss treatment outcome. Int J Obes Relat Metab Disord 1999;23:485-493.

95. Bulik CM, Reichborn-Kjennerud T. Medical morbidity in binge eating disorder. Int J Eat Disord 2003;34(Suppl):S39-S46.

96. Bulik CM, Sullivan PF, Kendler KS. Medical and psychiatric morbidity in obese women with and without binge eating. Int J Eat Disord 2002;32:72-78.

97. Tanofsky-Kraff M, Yanovski S. Eating disorder or disordered eating? Non-normative eating patterns in obese individuals. Obes Res 2004;12:1361-1366.

98. Hrabosky JI, Masheb RM, White MA, Grilo CM. Overvaluation of shape and weight in binge eating disorder. J Consult Clin Psychol 2007;75:175-180.

99. Mond JM, Hay PJ, Rodgers B, Owen C. Recurrent binge eating with and without the "undue influence of weight or shape on self-evaluation": Implications for the diagnosis of binge eating disorder. Behav Res Ther 2007;45:929-938.

100. Cachelin FM, Striegel-Moore RH, Elder KA, Pike KM, Wilfley DE, Fairburn CG. Natural course of a community sample of women with binge eating disorder. Int J Eat Disord 1999; 25:45-54

101. Fairburn CG, Cooper Z, Doll HA, Norman P, O'Connor M. The natural course of bulimia nervosa and binge eating disorder in young women. Arch Gen Psychiatry 2000;57:659-665.

102. Wilson GT, Vitousek KM, Loeb KL. Stepped-care treatment for eating disorders. J Consult Clin Psychol 2000;68:564-572.

103. Grilo CM. Guided self-help for binge eating disorder. In: Latner JD, Wilson GT, editors. Self-Help for Obesity and Eating Disorders. New York: Guilford Press, 2007, pp. 73-91.

104. Clyne C, Latner JD, Blampied N. Evaluation of a treatment programme for binge eating disorder (BED) teaching affect discrimination and regulation. Australian and New Zealand Academy for Eating Disorders Conference, Sydney, Australia, 2005. 